\title{
Assessment of incentivizing effects for cancer care frameworks
}

\author{
Jörg Haier ${ }^{1,4}$. Jonathan Sleeman ${ }^{2,3}$. Jürgen Schäfers ${ }^{4}$
}

Published online: 17 June 2020

(c) The Author(s) 2020, corrected publication 2021

\section{Importance of incentives for cancer care}

A central goal for healthcare systems worldwide is to provide sufficient cancer care to the population. However, it represents a major hurdle in many low- and middle-income countries (LMICs), resulting in an unnecessarily high proportion of patients with metastatic and advanced tumor stages. For many of these countries, significant differences between regions, between rural and urban populations, and across social groups remain major challenges for the reorganization of existing cancer care delivery, and the implementation of new structures that enable basic equal access to cancer care for the entire population. In our editorial series about incentives for cancer care in LMICs we have outlined how healthcare development in LMICs can be supported and guided by setting the right incentives. These encourage and steer the involved stakeholders towards improving the availability, acceptability, affordability and accessibility of sufficient quality of care, particularly for vulnerable populations such as those with metastatic disease. Financial as well as sociocultural incentives can motivate participants in the cancer care system (individuals, entities as well as organizations) to implement and carry out specific measures. However, the effects that are achieved are dependent on the healthcare framework. Setting incentives is frequently associated with potential conflicts of interests (CoI) that can potentially harm patients. When CoI and other forms of

Jörg Haier

haier.joerg@mh-hannover.de

1 Comprehensive Cancer Center, Hannover Medical School, Hannover, Germany

2 Medical Faculty Mannheim, University of Heidelberg, CBTM, Ludolf-Krehl-Str. 13 - 17, 68167 Mannheim, Germany

3 Institut für Toxikologie Und Genetik, Karlsruhe Institute for Technology (KIT), Campus Nord, Postfach 3640, 76021 Karlsruhe, Germany

4 IGP-Institute for Health Sciences and Public Health, Muenster, Germany decisional bias are sufficiently excluded or controlled, the intentional use of various types of incentives can promote beneficial cancer care development [1]. These incentives are not limited to physicians, and can impact on many stakeholders and professions, even including patients themselves, their relatives, as well as healthcare politicians.

Key financial incentives, their specific attributes and their potential impacts are summarized in Table 1 . The incentives include different financial models for reimbursement, such as fixed salaries, capitation, fee for service, or performancebased schemes, and can exert specific effects on cancer care systems. However, different targets within cancer care systems (for example the population covered by the cancer care system, risk selection, the number of treated cases, and the efficiency of resource usage) are influenced in different ways. Therefore, special attention needs to be paid when establishing effective incentive frameworks for the management of cancer patients. These incentive frameworks need to take into account the vulnerability of patients with advanced cancer, the often life-threatening course of their disease, as well as their dependence on costly diagnosis and treatment. In view of this, we suggest that for LMIC it may be beneficial to separate cancer care from other healthcare areas with regard to financial incentives and reimbursement schemes [2].

The allocation of qualitatively and quantitatively sufficient human resources to cancer care in LIMCs demands that sociocultural incentives are also understood and strategically used [3]. Similar to financial incentives, factors that influence that motivation of cancer care providers are intensively related to successful establishment of delivery models and the removal of implementation barriers. The entire cancer care community including all professionals groups, providers, organizations and policy makers therefore needs to balance the various types of incentives for each category of participant in the cancer care system. Furthermore, in order to guide the use of incentives in a dynamic manner, their effects need to be regularly assessed. 


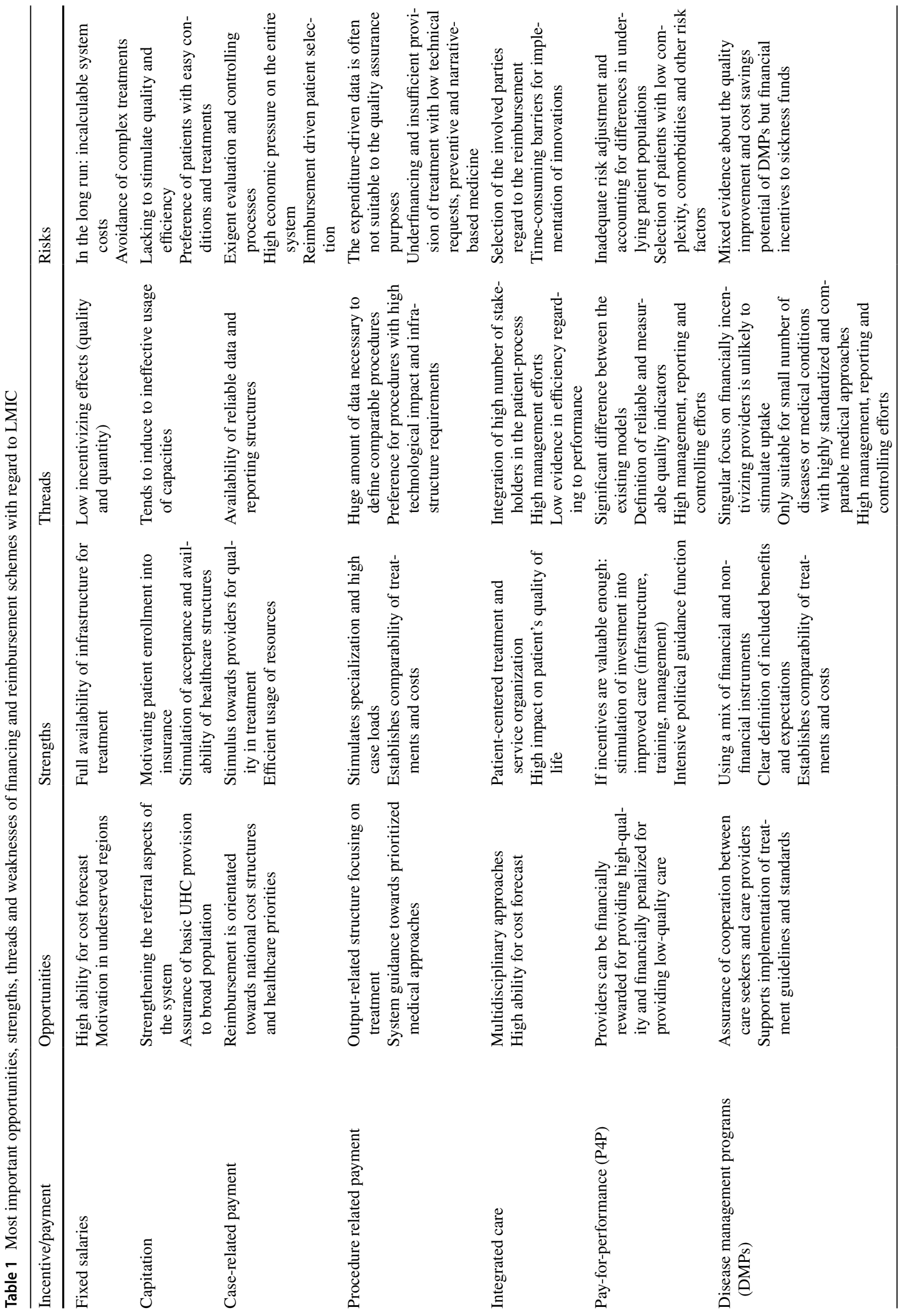




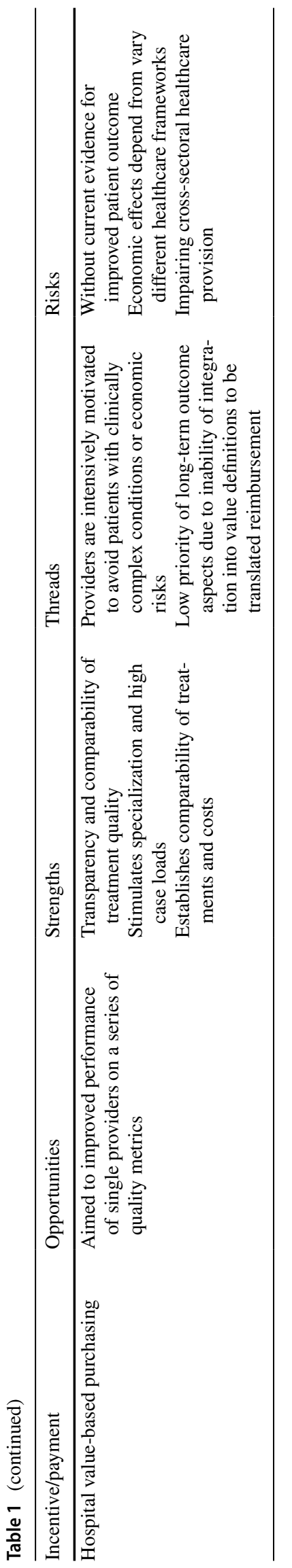

Assessment of incentivizing effects of cancer care framework

Incentivizing effects are always present in cancer care systems. They are heavily influenced by the political, economic, legal and sociocultural frameworks that are prevalent in each country. The resulting effects are dynamic, and differ between the various sociocultural environments. Therefore, due to rapidly changing societies, economic developments and prioritized efforts for Universal Health Coverage (UHC) in LMICs, awareness and consideration of incentivizing effects within the given healthcare framework is crucial not only for successful implementation but also for achievement of the expected outcomes. Awareness and anticipation of incentivizing effects need to be ensured before novel framework conditions are set in place. In addition, incentivizing effects need to be continuously evaluated and supervised during and after implementation of these changes. Evaluations have to cover the individual, organizational and systemic levels, and include all major stakeholders and UHC aspects. As far as possible, the performance of any cancer care system should be assessed with respect to the scope and quality of services actually received by patients.

Before implementation of incentivizing measures, such as financial incentives, reimbursement schemes and additional economic incentives, a number of parameters need to be carefully considered, and their potential and expected consequences need to be continuously assessed. For example, impacts on the covered population, the number of treated cases, and the number and complexity of procedures need to be estimated. Effects on risk selection, the accessibility of cancer care structures, and possible barriers for cancer care use should be appraised. In addition, the consequences for the clinical quality of cancer care and the efficient use of resources need to be gauged.

During and after implementation, there needs to be continuous supervision and assessment of the effects that are realized. To this end, predefined indicators need to be implemented to structure the ongoing measurement and quantification of the incentivizing effects.

Evaluation of the impact of sociocultural incentives needs to be carefully designed, as these may be a direct component of novel healthcare frameworks, but can also have indirect effects on cancer care delivery processes. Key areas for consideration include professional education (including supervision and training), career development, and professional migration within the system, both nationally (rural, remote, urban) and internationally (loss and/or gain of healthcare professionals and knowledge). In addition, a number of social aspects have important incentivizing effects that need to be monitored, including 
housing, volunteer motivation, social recognition and community appreciation. The motivational impact of sociocultural incentives provided by the available infrastructure (including human resource environment) needs to be evaluated, as do innovations and delivery processes for various healthcare professional groups that enable them to provide a high quality of care.

For all analytical steps it is necessary to predefine a catalogue of indicators that allow the evaluation parameters outlined above to be measured. These may differ between countries and healthcare systems, and can depend on priority definitions and cultural value systems. Indeed, the evaluation of incentivizing effects always need to take into account the general aim of UHC, and to identify potential misuse of these incentives as inappropriate CoI.

In summary, different types of incentives have varying effects on the successful implementation of cancer care in LMICs, which can be particularly decisive for the effective management of patients with metastatic disease. The results of incentives can be stimulating, but can also build up additional barriers for healthcare system development. Their impact is subject to constant change, and depends on the prevailing environmental framework. For all types of incentives, transparency, public discussion and strategic implementation should be considered as a prerequisite for achieving progress towards UHC, in particular for the implementation of full cancer care coverage for all populations in LMICs, and especially for those patients with metastatic disease.
Funding Open Access funding enabled and organized by Projekt DEAL.

Open Access This article is licensed under a Creative Commons Attribution 4.0 International License, which permits use, sharing, adaptation, distribution and reproduction in any medium or format, as long as you give appropriate credit to the original author(s) and the source, provide a link to the Creative Commons licence, and indicate if changes were made. The images or other third party material in this article are included in the article's Creative Commons licence, unless indicated otherwise in a credit line to the material. If material is not included in the article's Creative Commons licence and your intended use is not permitted by statutory regulation or exceeds the permitted use, you will need to obtain permission directly from the copyright holder. To view a copy of this licence, visit http://creativecommons.org/licenses/by/4.0/.

\section{References}

1. Haier J, Sleeman J, Schäfers J (2019) Cancer care in low- and middle-income countries. Clin Exp Metastasis 36(6):477-480. https://doi.org/10.1007/s10585-019-10003-4

2. Haier J, Sleeman J, Schäfers J (2020) Guidance of healthcare development for metastatic cancer patients as an example for setting incentives. Clin Exp Metastasis 37(1):1-5. https://doi.org/10. 1007/s10585-019-10014-1

3. Haier J, Sleeman J, Schäfers J (2020) Sociocultural incentives for cancer care implementation. Clin Exp Metastasis. in press

Publisher's Note Springer Nature remains neutral with regard to jurisdictional claims in published maps and institutional affiliations. 